Supporting Information

\title{
Electrochemical Oxidation of Hexafluoropropylene Oxide Dimer Acid (GenX): Mechanistic Insights and Efficient Treatment Train with Nanofiltration
}

Nasim E. Pica ${ }^{1}$, Joanna Funkhouser ${ }^{1}$, Yiming Yin $^{1}$, Zuoyou Zhang ${ }^{1}$, Donato M. Ceres ${ }^{2}$, Tiezheng Tong ${ }^{1}$, Jens Blotevogel ${ }^{1, *}$

${ }^{1}$ Department of Civil and Environmental Engineering, Colorado State University, Fort Collins, CO 80523, USA

${ }^{2}$ Advanced Diamond Technologies, Inc., 48 East Belmont Drive, Romeoville, IL 60446, USA

*CORRESPONDING AUTHOR:

Jens Blotevogel, Colorado State University, Department of Civil and Environmental Engineering, 1320 Campus Delivery, Fort Collins, Colorado 80523-1320, USA.

Email: jens.blotevogel@colostate.edu; Phone: +1-970-491-8880

Number of Pages: 10; Number of Figures: 3 


\section{Alternative Oxidative Decomposition Reactions}

(3) $\mathrm{C}_{3} \mathrm{~F}_{7} \mathrm{OCFCF}_{3} \mathrm{OH}$
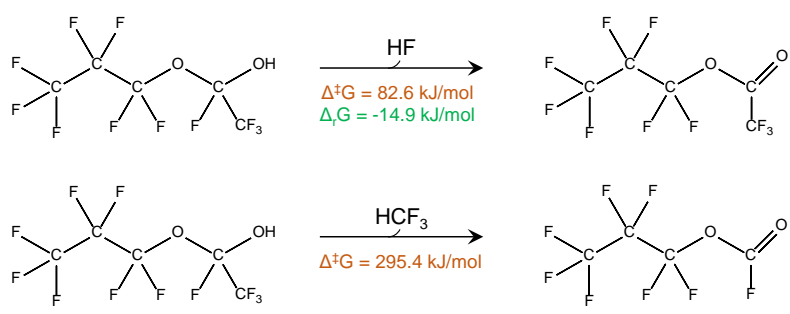

(4) $\mathrm{C}_{3} \mathrm{~F}_{7} \mathrm{OCFCF}_{3} \mathrm{O} \cdot$
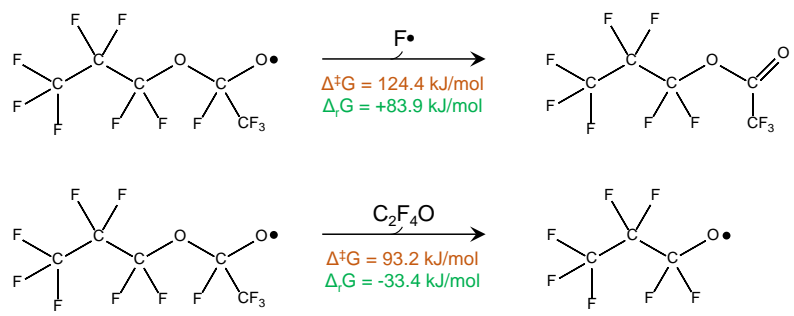

(5) $\mathrm{C}_{3} \mathrm{~F}_{7} \mathrm{OCFO}$
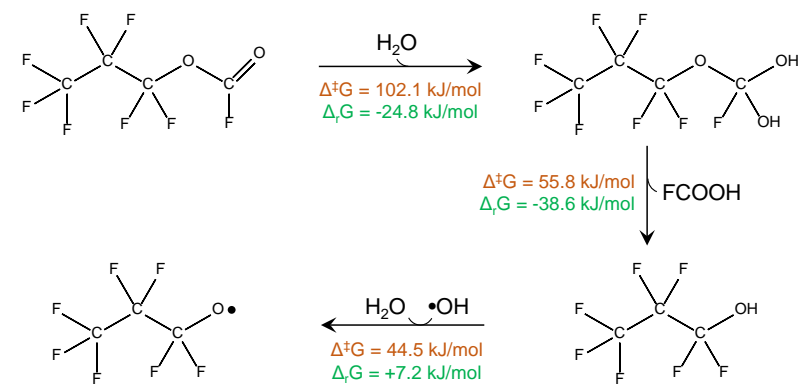

(7) $\mathrm{C}_{3} \mathrm{~F}_{7} \mathrm{OCF}(\mathrm{OH}) \mathrm{O} \cdot$

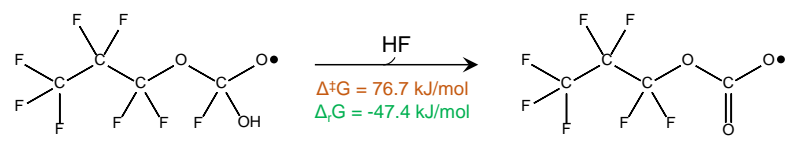

(8) $\mathrm{C}_{3} \mathrm{~F}_{7} \mathrm{O} \cdot$
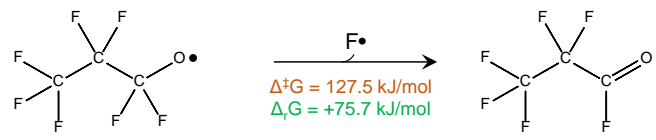

Figure S1. Kinetically less favorable oxidative decomposition reactions for HFPO-DA intermediates based on DFT calculations. 


\section{Operational Regime of Electrochemical HFPO-DA Oxidation}

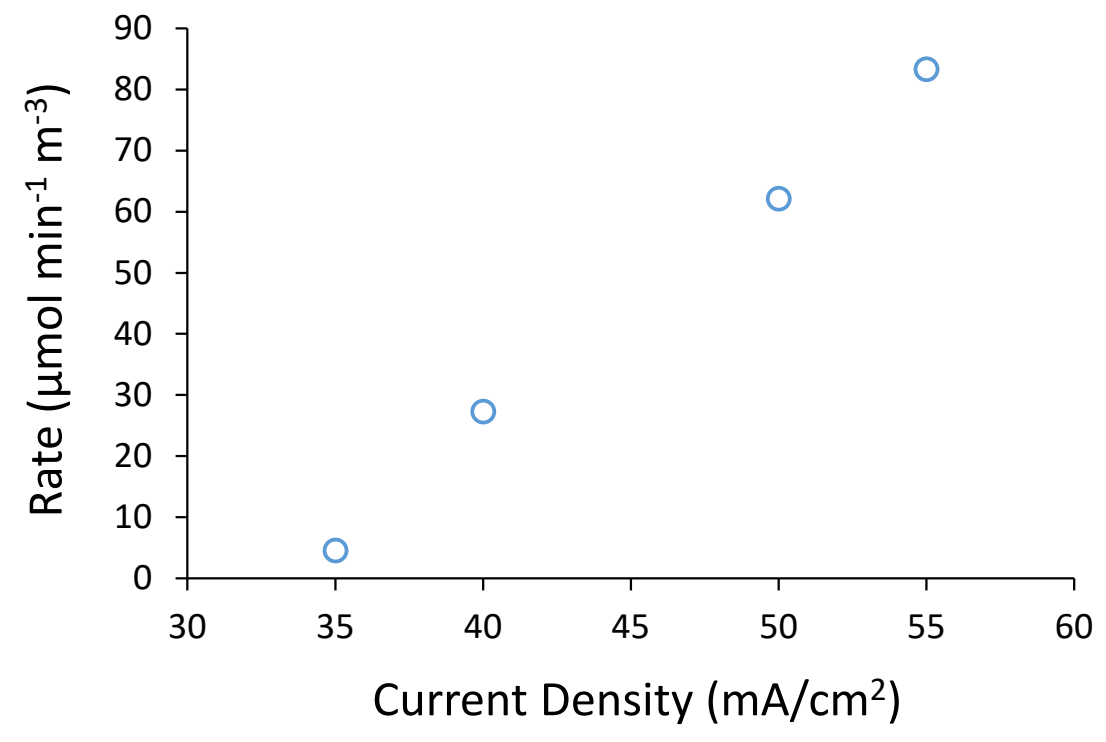

Figure S2. Electrochemical HFPO-DA oxidation rate as a function of current density in the nanofiltration rejectate $\left(2475 \mathrm{mg} / \mathrm{L} \mathrm{Na}_{2} \mathrm{SO}_{4}, 495 \mathrm{mg} / \mathrm{L} \mathrm{NaCl}\right)$. The observed increase in rate with increasing current density implies current-limited conditions at the current density of $50 \mathrm{~mA} / \mathrm{cm}^{2}$ applied in the experiments. 


\section{Effect of Methanol Quenching on the Electrooxidation of HFPO-DA}

To further probe the contribution of electrochemically activated sulfate to the electrochemical oxidation of HFPO-DA, a radical quenching experiment was conducted in the nanofiltration rejectate. In the absence of $\mathrm{SO}_{4}{ }^{-}$-selective radical probes (ref. 45 in the main manuscript), methanol was chosen as radical quencher at a concentration of $500 \mathrm{mg} / \mathrm{L}(15.6 \mathrm{mM})$, roughly equimolar to the sulfate concentration of $17.4 \mathrm{mM}$. While methanol has a similar reactivity to ${ }^{\circ} \mathrm{OH}$ and $\mathrm{SO}_{4}{ }^{-}$, our theoretical investigations suggested that ${ }^{\circ} \mathrm{OH}$ is unreactive towards HFPO-DA. Thus, any decrease in the observed electrochemical oxidation rate was rationalized to be due to suppressing the contributions of $\mathrm{SO}_{4}{ }^{-{ }^{-}}$to HFPO-DA oxidation.

Figure S3 illustrates that the addition of methanol indeed lowered the observed electrochemical HFPODA oxidation rate constant from $0.0041 \mathrm{~min}^{-1}$ to $0.0022 \mathrm{~min}^{-1}$, similar to the observed rate in the lowsulfate control experiment $\left(0.0019 \mathrm{~min}^{-1}\right)$. We acknowledge, however, that under current-controlled conditions as operated here we cannot fully exclude oxidation of methanol through direct electron transfer, thus possibly directly competing with HFPO-DA oxidation at the anode surface.

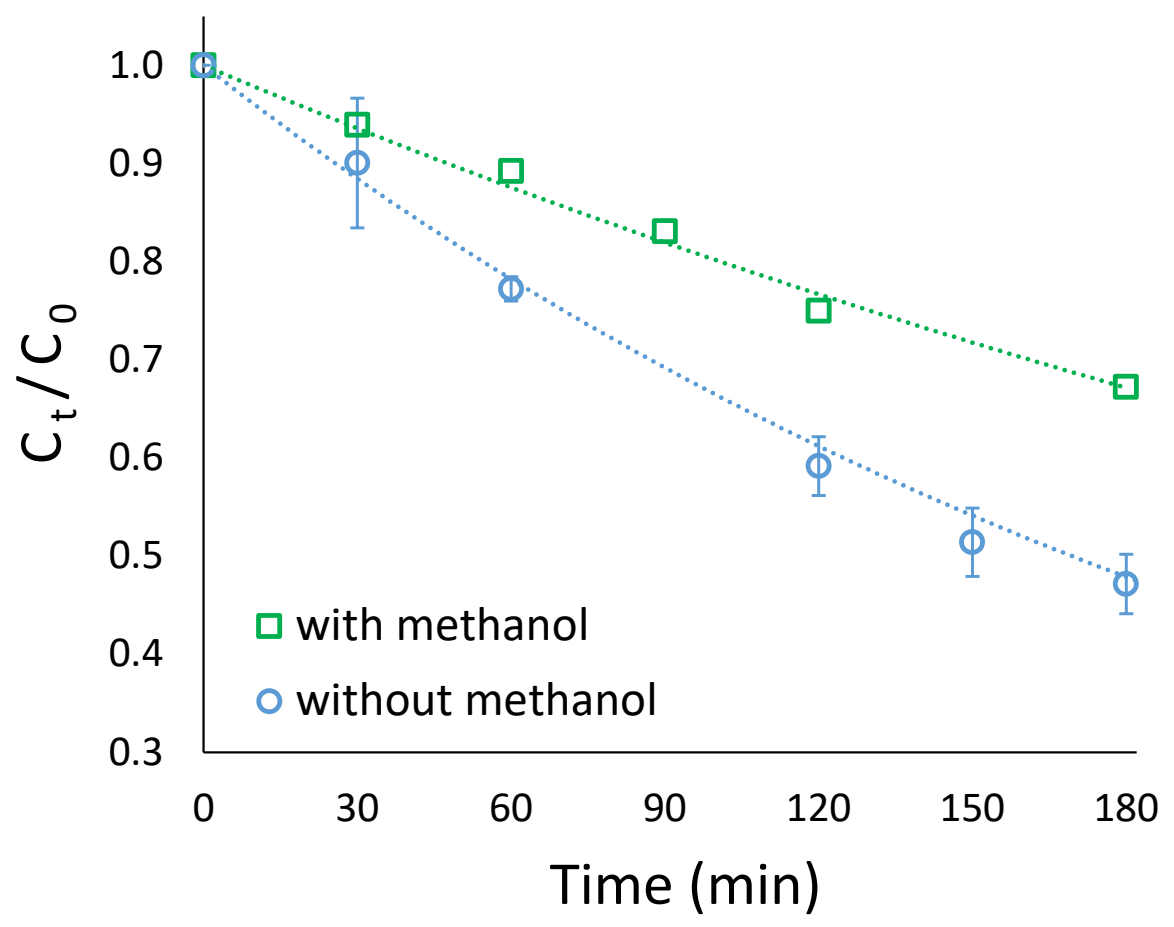

Figure S3. Pseudo-first-order kinetics for electrochemical oxidation of HFPO-DA in the nanofiltration rejectate (2475 mg/L Na $2 \mathrm{SO}_{4}, 495 \mathrm{mg} / \mathrm{L} \mathrm{NaCl}, 50 \mathrm{~mA} / \mathrm{cm}^{2}$ ) in the absence (blue) and presence (green) of $500 \mathrm{mg} / \mathrm{L}$ methanol. 


\section{Optimized Structures of Ground States, Intermediates, and Transition States for the Proposed Oxidative Decomposition Pathway in Figure 1}

(1)

$\begin{array}{lrrr}\text { C } & -4.16083900 & -0.06540100 & -0.48620800 \\ \text { C } & -3.27880300 & -1.23396900 & -0.00386600 \\ \text { C } & -1.77500100 & -0.90331800 & 0.11826200 \\ \text { C } & 0.22366100 & -2.16690200 & 0.66421600 \\ \text { C } & 0.48484300 & -3.50681100 & 1.48734000 \\ \text { O } & 0.82436200 & -4.45867900 & 0.78936900 \\ \text { O } & 0.29070100 & -3.34901200 & 2.69581300 \\ \text { F } & 0.98713800 & 0.07752400 & 0.41570800 \\ \text { F } & 0.46187700 & -0.54466500 & 2.40631500 \\ \text { F } & 0.63881800 & -2.25388400 & -0.62219700 \\ \text { F } & -1.31783300 & -0.59436100 & -1.10716400 \\ \text { F } & -1.66508300 & 0.21920400 & 0.86266900 \\ \text { F } & -3.75837100 & -1.61060000 & 1.18662200 \\ \text { F } & -3.43486900 & -2.23354700 & -0.88111800 \\ \text { F } & -3.73024000 & 0.42500300 & -1.64120500 \\ \text { F } & -4.19750600 & 0.91207700 & 0.40882100 \\ \text { F } & -5.40464900 & -0.51146000 & -0.66203500 \\ \text { O } & -1.18489500 & -1.96736200 & 0.67658600 \\ \text { C } & 0.97729500 & -0.97067500 & 1.26446700 \\ \text { F } & 2.25132200 & -1.30682100 & 1.46777700\end{array}$

(2)

$\begin{array}{lrrc}\text { C } & -4.28087473 & 0.00032599 & -0.53088289 \\ \text { C } & -3.34803628 & -1.14107941 & -0.07934667 \\ \text { C } & -1.87200036 & -0.72958175 & 0.07525617 \\ \text { C } & 0.14570950 & -1.87981694 & 0.45559993 \\ \text { F } & 0.98915482 & 0.32486449 & 0.44556255 \\ \text { F } & 0.57449396 & -0.60521447 & 2.34802240 \\ \text { F } & 0.58933076 & -2.25794614 & -0.72499795 \\ \text { F } & -1.37229990 & -0.35436544 & -1.10092888 \\ \text { F } & -1.77221924 & 0.30002898 & 0.91584467 \\ \text { F } & -3.79081356 & -1.59181344 & 1.09744928 \\ \text { F } & -3.41622509 & -2.11606389 & -0.98975488 \\ \text { F } & -3.86233280 & 0.51296017 & -1.67833066 \\ \text { F } & -4.32171928 & 0.95515731 & 0.38509887 \\ \text { F } & -5.49967676 & -0.49065196 & -0.69611877 \\ \text { O } & -1.19726729 & -1.82957518 & 0.57031816 \\ \text { C } & 1.00543820 & -0.83325752 & 1.11514631 \\ \text { F } & 2.26284496 & -1.25580073 & 1.15866796\end{array}$




\section{(3)}

$\begin{array}{lrcc}\text { C } & -4.07555082 & 0.04318802 & -0.49357671 \\ \text { C } & -3.28298326 & -1.19930287 & -0.04055728 \\ \text { C } & -1.78089169 & -0.94540324 & 0.20057489 \\ \text { C } & 0.09894059 & -2.30247567 & 0.66817337 \\ \text { F } & 0.18383969 & -2.78149167 & 2.98243986 \\ \text { F } & -0.27246900 & -4.41440548 & 1.64972867 \\ F & 0.76532592 & -1.15763115 & 0.96032134 \\ \text { F } & -1.19456920 & -0.60326532 & -0.94697611 \\ \text { F } & -1.63584707 & 0.07102945 & 1.05555849 \\ \text { F } & -3.83437674 & -1.63979599 & 1.09449420 \\ \text { F } & -3.40243465 & -2.13351661 & -0.98841300 \\ \text { F } & -3.53755801 & 0.56648708 & -1.58594941 \\ \text { F } & -4.09636895 & 0.95772299 & 0.46312808 \\ F & -5.32027662 & -0.32533882 & -0.76103542 \\ \text { O } & -1.26794019 & -2.10310136 & 0.71804370 \\ \text { C } & 0.42857643 & -3.30245471 & 1.79381194 \\ \text { F } & 1.72846443 & -3.60457940 & 1.71322075 \\ \text { O } & 0.45204546 & -2.77200431 & -0.55229121 \\ \text { H } & 1.40449824 & -2.92165002 & -0.57655338\end{array}$

\section{TS (3) $\rightarrow$ (4)}

$\begin{array}{lrrr}\text { C } & -4.01150833 & 0.03967183 & -0.48171131 \\ \text { C } & -3.24538601 & -1.20899328 & -0.00139151 \\ \text { C } & -1.72984151 & -0.99517937 & 0.18971402 \\ \text { C } & 0.13201018 & -2.39387547 & 0.64500999 \\ \text { F } & 0.16422223 & -2.82222475 & 2.96405544 \\ \text { F } & -0.29414177 & -4.47579450 & 1.65367513 \\ \text { F } & 0.83700265 & -1.26734808 & 0.87411592 \\ \text { F } & -1.16679839 & -0.71120859 & -0.98613804 \\ \text { F } & -1.52911571 & 0.04589388 & 1.00230982 \\ \text { F } & -3.77851929 & -1.58768414 & 1.16407405 \\ \text { F } & -3.41977818 & -2.17483885 & -0.90867734 \\ \text { F } & -3.48237448 & 0.50891987 & -1.60242952 \\ \text { F } & -3.98577150 & 0.98640324 & 0.44288368 \\ \text { F } & -5.27163080 & -0.30164906 & -0.71062285 \\ \text { O } & -1.23841862 & -2.14913975 & 0.73534568 \\ \text { C } & 0.43616636 & -3.37903834 & 1.79439622 \\ \text { F } & 1.72059600 & -3.71811611 & 1.77001391 \\ \text { O } & 0.40039847 & -2.91583292 & -0.57481412 \\ \text { H } & 1.50377304 & -2.70046644 & -0.95882552 \\ \text { O } & 2.61243583 & -2.84352070 & -0.79259805 \\ \text { H } & 2.76462636 & -3.80050424 & -0.81143577\end{array}$

\section{(4)}

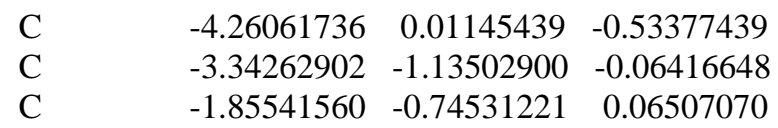




$\begin{array}{llcc}\text { C } & 0.16538034 & -1.99779842 & 0.50916702 \\ \text { F } & 0.87826916 & 0.24986605 & 0.39611383 \\ \text { F } & 0.53506301 & -0.56664952 & 2.36518011 \\ \text { F } & 0.54336272 & -2.08154452 & -0.78106775 \\ \text { F } & -1.38227106 & -0.39351865 & -1.12855495 \\ \text { F } & -1.74466498 & 0.30930006 & 0.88013462 \\ \text { F } & -3.78579046 & -1.54943925 & 1.12593738 \\ \text { F } & -3.43798349 & -2.13002750 & -0.95097259 \\ \text { F } & -3.84593929 & 0.49552202 & -1.69489254 \\ \text { F } & -4.28214232 & 0.98544498 & 0.36288241 \\ \text { F } & -5.48803459 & -0.46533539 & -0.67957033 \\ \text { O } & -1.21619919 & -1.83733969 & 0.59079016 \\ \text { C } & 0.98780848 & -0.82784116 & 1.15354757 \\ \text { F } & 2.26195864 & -1.17627466 & 1.22359477 \\ \text { O } & 0.48718681 & -3.09121175 & 1.21197076\end{array}$

\section{TS (4) $\rightarrow$ (5)}

$\begin{array}{lccc}\text { C } & -4.25944081 & 0.01208913 & -0.56808735 \\ \text { C } & -3.36660239 & -1.13133854 & -0.04535753 \\ \text { C } & -1.86792924 & -0.77555652 & 0.05882061 \\ \text { C } & 0.09288536 & -2.20917798 & 0.40193017 \\ \text { F } & 0.88722695 & 0.30733705 & 0.50880518 \\ \text { F } & 0.55752483 & -0.58783722 & 2.45167741 \\ \text { F } & 0.51150520 & -1.96845478 & -0.84757793 \\ \text { F } & -1.41785835 & -0.39308888 & -1.13294345 \\ \text { F } & -1.72200233 & 0.25188563 & 0.90396937 \\ \text { F } & -3.80885051 & -1.47467103 & 1.16728771 \\ \text { F } & -3.49633629 & -2.16579381 & -0.88140565 \\ \text { F } & -3.89838526 & 0.36034363 & -1.79397552 \\ \text { F } & -4.17725398 & 1.06786504 & 0.22742583 \\ \text { F } & -5.51421389 & -0.41131089 & -0.59304692 \\ \text { O } & -1.24405424 & -1.88979352 & 0.55519641 \\ \text { C } & 1.06732389 & -0.74306785 & 1.26602641 \\ \text { F } & 2.31754915 & -1.09168857 & 1.30323800 \\ \text { O } & 0.55625249 & -3.10347631 & 1.06940790\end{array}$

(5)

$\begin{array}{lrrc}\text { C } & -4.23869084 & 0.05112018 & -0.52933421 \\ \text { C } & -3.40826530 & -1.15916421 & -0.05636237 \\ \text { C } & -1.88169633 & -0.94825347 & -0.11352910 \\ \text { C } & 0.02168463 & -2.27164150 & 0.42396675 \\ \text { F } & 0.65737568 & -1.19128877 & 0.00865405 \\ \text { F } & -1.51176483 & -0.68445377 & -1.36540590 \\ \text { F } & -1.53976119 & 0.08042721 & 0.65931862 \\ \text { F } & -3.75034893 & -1.42213085 & 1.20784424 \\ \text { F } & -3.71843112 & -2.19951943 & -0.83406762 \\ \text { F } & -3.95781429 & 0.34441769 & -1.78948216 \\ \text { F } & -3.99388054 & 1.10757893 & 0.23029890 \\ \text { F } & -5.52237084 & -0.26038155 & -0.43447043\end{array}$


$\begin{array}{lrrr}\text { O } & -1.32023994 & -2.12334610 & 0.33918578 \\ O & 0.54288126 & -3.24073469 & 0.81588102\end{array}$

(6)

$\begin{array}{lccc}\text { C } & -4.30392713 & 0.04787446 & -0.53181862 \\ \text { C } & -3.42486653 & -1.13668826 & -0.06122364 \\ \text { C } & -1.91572963 & -0.81994530 & -0.15593873 \\ \text { C } & -0.03282011 & -2.22927674 & 0.40043546 \\ \text { F } & 0.78282220 & -1.32801691 & 0.05800567 \\ F & -1.47923898 & -0.64756810 & -1.36475751 \\ \text { F } & -1.51166473 & 0.11606942 & 0.64499273 \\ \text { F } & -3.68659882 & -1.41503846 & 1.21090600 \\ F & -3.64836747 & -2.19718216 & -0.82931341 \\ \text { F } & -4.00689127 & 0.32890494 & -1.78896584 \\ F & -4.05215531 & 1.09780549 & 0.23080081 \\ \text { F } & -5.56166612 & -0.30344159 & -0.42595668 \\ \text { O } & -1.27177325 & -2.11464192 & 0.34801891 \\ \text { O } & 0.49155197 & -3.31622627 & 0.82731232\end{array}$

\section{TS (5) $\rightarrow$ (7)}

$\begin{array}{lrrr}\text { C } & -4.15410682 & -0.04067413 & -0.74778992 \\ \text { C } & -3.40948478 & -1.02138394 & 0.17936585 \\ \text { C } & -1.97687435 & -1.37107123 & -0.27339533 \\ \text { F } & 0.40165529 & -1.23425438 & 1.26299298 \\ \text { F } & -2.02294828 & -1.95010921 & -1.47262684 \\ \text { F } & -1.25839227 & -0.25484411 & -0.38129020 \\ \text { F } & -3.34488703 & -0.46190357 & 1.39217966 \\ \text { F } & -4.12212090 & -2.14930261 & 0.24353127 \\ \text { F } & -4.22939059 & -0.52422196 & -1.97831794 \\ \text { F } & -3.53536648 & 1.13029808 & -0.77573142 \\ \text { F } & -5.38088557 & 0.13419231 & -0.27865101 \\ \text { O } & -1.47759238 & -2.24960850 & 0.65672903 \\ \text { O } & 0.32399428 & -3.44414900 & 1.23669992 \\ \text { C } & -0.14435920 & -2.38505255 & 0.89563910 \\ \text { O } & 0.54973417 & -2.58403071 & -0.78342216 \\ \text { H } & 1.50648744 & -2.56325612 & -0.60676911\end{array}$

\section{TS (6) $\rightarrow$ (7)}

$\begin{array}{lrrr}\text { C } & -4.12088900 & -0.11816800 & 0.23178000 \\ \text { C } & -2.91779600 & -0.93363000 & 0.75352300 \\ \text { C } & -1.78431800 & -1.10381700 & -0.27959100 \\ \text { C } & 0.28665300 & -2.24646800 & -0.32471500 \\ \text { F } & 0.38449800 & -1.69858400 & -1.54107400 \\ \text { F } & -2.22074300 & -1.83671300 & -1.32145500 \\ F & -1.38856100 & 0.07026000 & -0.75311400 \\ \text { F } & -2.41709000 & -0.31564000 & 1.82001300\end{array}$




$\begin{array}{lrrr}\text { F } & -3.34075000 & -2.15528700 & 1.08601200 \\ \text { F } & -4.57907700 & -0.66519300 & -0.88883700 \\ \text { F } & -3.75037900 & 1.12708300 & -0.01255900 \\ \text { F } & -5.06732600 & -0.12936700 & 1.14594100 \\ \text { O } & -0.75090600 & -1.75420300 & 0.37014300 \\ \text { O } & 1.24614900 & -2.74983800 & 0.21921100 \\ \text { O } & -0.20391700 & -3.91587200 & -0.87432200 \\ \text { H } & -0.35027800 & -4.42137600 & -0.05393100 \\ \mathrm{O} & -0.97150400 & -1.16685400 & -3.99494100 \\ \mathrm{H} & 0.35496500 & -1.73794700 & -4.15651900 \\ \mathrm{H} & -1.57736300 & -1.08385100 & -4.73742200 \\ \mathrm{O} & 1.31151300 & -2.11816900 & -4.27901800 \\ \mathrm{H} & 1.96683600 & -1.41982500 & -4.15729300 \\ \mathrm{H} & 1.52207700 & -2.95680800 & -3.64270200 \\ \mathrm{O} & 1.82870700 & -4.01246000 & -2.83354200 \\ \mathrm{H} & 2.12189800 & -4.83857500 & -3.22966700 \\ \mathrm{H} & 1.22246200 & -4.21047400 & -2.10110900 \\ \mathrm{H} & -1.49033200 & -1.31305700 & -3.19659700\end{array}$

(7)

$\begin{array}{lrcc}\text { C } & -4.16972330 & -0.05811723 & -0.74316097 \\ \text { C } & -3.37950826 & -1.01088391 & 0.17660923 \\ \text { C } & -1.94581224 & -1.31623565 & -0.30410448 \\ \text { F } & 0.41788487 & -1.46509781 & 1.38726012 \\ \text { F } & -1.98846338 & -1.71954255 & -1.57828557 \\ \text { F } & -1.21627476 & -0.19847449 & -0.25335077 \\ \text { F } & -3.30322867 & -0.44631370 & 1.38607593 \\ F & -4.06541025 & -2.15475515 & 0.26124570 \\ \text { F } & -4.43892221 & -0.64111934 & -1.90055833 \\ \text { F } & -3.47958998 & 1.05204916 & -0.96426227 \\ \text { F } & -5.31139528 & 0.25656916 & -0.14775914 \\ \text { O } & -1.45376764 & -2.29507388 & 0.51133778 \\ \text { O } & 0.20261219 & -3.64617365 & 1.19073466 \\ \text { C } & -0.07877476 & -2.45378637 & 0.61113347 \\ \text { O } & 0.49190034 & -2.45873287 & -0.62227427 \\ \text { H } & 1.44959788 & -2.40524832 & -0.51691222\end{array}$

\section{TS (7) $\rightarrow(8)$}

$\begin{array}{llcc}\text { C } & -4.13868321 & -0.11322842 & -0.84123841 \\ \text { C } & -3.28043128 & -0.83893970 & 0.21702654 \\ \text { C } & -2.16224006 & -1.74099381 & -0.37878039 \\ \text { F } & 0.55051284 & -2.93440765 & 1.63745921 \\ \text { F } & -2.73485583 & -2.74391706 & -1.05082469 \\ F & -1.45379924 & -1.01613929 & -1.25728236 \\ \text { F } & -2.71692296 & 0.10081933 & 0.98693242 \\ F & -4.09164055 & -1.59473205 & 0.95998016 \\ F & -4.64713736 & -0.97526016 & -1.70708556 \\ \text { F } & -3.41544548 & 0.78551796 & -1.49065715 \\ F & -5.13226975 & 0.50816860 & -0.21934527\end{array}$




$\begin{array}{llll}\mathrm{O} & -1.42914174 & -2.17498029 & 0.66536288 \\ \mathrm{O} & -0.02386160 & -3.33950804 & -0.47873852 \\ \mathrm{C} & 0.29814104 & -2.52399090 & 0.39985345 \\ \mathrm{O} & 0.92202225 & -1.40164953 & 0.11828493 \\ \mathrm{H} & 1.18687400 & -0.95769720 & 0.93278353\end{array}$

(8)

$\begin{array}{lrrr}\text { C } & -4.12272342 & -0.08880444 & -0.84330542 \\ \text { C } & -3.30348617 & -0.83626619 & 0.23431975 \\ \text { C } & -2.18531683 & -1.74641347 & -0.37213551 \\ \text { F } & -2.75298982 & -2.80595813 & -0.96123030 \\ \text { F } & -1.52877110 & -1.06441533 & -1.32327557 \\ \text { F } & -2.75192951 & 0.07670910 & 1.03753386 \\ \text { F } & -4.13512586 & -1.60621634 & 0.93493943 \\ \text { F } & -4.54108611 & -0.93225190 & -1.77414986 \\ \text { F } & -3.39504613 & 0.86096543 & -1.40443756 \\ \text { F } & -5.17363898 & 0.46555436 & -0.25694114 \\ \text { O } & -1.39539139 & -2.10606162 & 0.64529604\end{array}$

\section{TS (8) $\rightarrow$ (9)}

$\begin{array}{llrr}\text { C } & -4.15160669 & -0.08799497 & -0.76409222 \\ \text { C } & -3.46771885 & -1.00837714 & 0.27143466 \\ \text { C } & -1.75420644 & -1.45749792 & -0.37300138 \\ \text { F } & -1.98020210 & -1.58468238 & -1.68499675 \\ \text { F } & -1.12654310 & -0.29400705 & -0.19051600 \\ \text { F } & -3.24329441 & -0.38140861 & 1.39659016 \\ \text { F } & -4.16363877 & -2.09834022 & 0.45086014 \\ \text { F } & -4.52743271 & -0.78915244 & -1.81511774 \\ \text { F } & -3.34635359 & 0.88823248 & -1.13473993 \\ \text { F } & -5.22935637 & 0.43637716 & -0.18800121 \\ \text { O } & -1.54595826 & -2.41904426 & 0.34216468\end{array}$

(9)

$\begin{array}{llll}\text { C } & -4.18164448 & -0.09011777 & -0.74799610 \\ \text { C } & -3.52049531 & -1.00691336 & 0.25421101 \\ \text { F } & -3.10459844 & -0.39383802 & 1.33747623 \\ \text { F } & -4.22268780 & -2.08071498 & 0.52981603 \\ \text { F } & -4.46616524 & -0.76377971 & -1.85385198 \\ \text { F } & -3.36991553 & 0.91711752 & -1.03832379 \\ \text { F } & -5.31829247 & 0.41507470 & -0.26222269\end{array}$

